\title{
Scolopendra Pharmacopuncture Combined with Electroacupuncture for the Treatment of Ganglion Cysts: A Retrospective Study
}

\author{
Seon Choe ${ }^{1,2 \dagger}$, Ui Min Jerng ${ }^{3 \dagger}$, Jeong Hwan Park ${ }^{2}$, Sungha Kim², Sungchul Kim ${ }^{4}$, Jinbok Lee ${ }^{5}$, \\ Jun-Hwan Lee ${ }^{2 *}$, Minseop Shin ${ }^{6} *$ \\ ${ }^{1}$ Department of Biomedical Sciences, Seoul National University Graduate School, Seoul, Republic of Korea \\ ${ }^{2}$ Clinical Medicine Division, Korea Institute of Oriental Medicine, Daejeon, Republic of Korea \\ ${ }^{3}$ Department of Internal Medicine, College of Korean Medicine, Sangji University, Wonju, Republic of Korea \\ ${ }^{4}$ Department of Acupuncture \& Moxibustion, Wonkwang University Gwangju Medical Hospital, Gwangju, Republic of Korea \\ ${ }^{5}$ Lee Jinbok Korean Medicine Clinic, Jeonju, Republic of Korea \\ ${ }^{6}$ Shin Min Seop Korean Medicine Clinic, Jeonju, Republic of Korea
}

Received September 10, 2020

Reviewed September 16, 2020

Accepted November 20, 2020

\section{*Corresponding Authors}

\section{Minseop Shin}

Shin Min Seop Korean Medicine Clinic, Jeonju 55081, Republic of Korea

Tel: +82-63-228-7579

E-mail: kahn815@hanmail.net

Jun-Hwan Lee

Clinical Medicine Division, Korea Institute of Oriental Medicine, Daejeon 34054, Republic of Korea

Tel: +82-42-868-9693

E-mail: omdjun@kiom.re.kr

${ }^{\dagger}$ These authors contributed equally to this work.
Objectives: Ganglion cysts require a sustainable treatment that suppresses their frequent recurrence. This study aimed to explore the clinical effects of Scolopendra pharmacopuncture (SP) and electroacupuncture on ganglion cysts.

Methods: We retrospectively reviewed the patient records and follow-up reports for 20 patients with wrist ganglion cysts who received SP and electroacupuncture from April 2016 to March 2017. The cyst diameter, recurrence, visual analog scale (VAS) scores for pain, the Korean version of the disabilities of arm, shoulder, and hand (K-DASH) score, and the Korean version of the patient-rated wrist evaluation (K-PRWE) score before and after treatment were noted.

Results: After treatment, the cyst diameter decreased significantly from $13.61 \pm 6.41 \mathrm{~mm}$ to $5.15 \pm 6.18 \mathrm{~mm}(\mathrm{p}<0.001)$, and VAS score for pain decreased from $1.31 \pm 1.77$ to 0.41 $\pm 0.33(p=0.021)$. Further, the K-DASH score decreased significantly from $8.97 \pm 12.66$ to $2.21 \pm 7.39(p=0.016)$, and score for the function subscale of K-PRWE decreased from $11.37 \pm 4.48$ to $9.1 \pm 3.67(p=0.046)$. No recurrences were reported from the followedup patients. Any complication related to SP or electroacupuncture was not observed, except mild rash, itching, and swelling at the injection site in four patients.

Conclusion: Combination of SP and electroacupuncture may be effective in treating ganglion cysts; further prospective studies with large population are needed to clarify the effect of SP and electroacupuncture.

Keywords: scolopendra pharmacopuncture, electroacupuncture, ganglion cysts, retrospective studies

\section{INTRODUCTION}

Ganglion cyst is one of the most common soft tissue masses found in the synovial tissue $[1,2]$ These cysts may develop in any connective tissue, such as the joint capsules and tendon sheaths [3]. Most ganglion cysts are asymptomatic; however, some patients experience pain, tenderness, weakness, and abnormal sensation depending on the size and location of the le- sion [4]. Patients with ganglion cysts are typically educated and reassured regarding the mass; no further intervention is usually suggested, or they are offered either aspiration, often combined with the injection of various substances, or surgical excision [1]. Generally, aspiration, followed by steroid injection is the most popular treatment. Aspiration is considered safe and associated with a low risk of local skin infection; however, it could cause increased pain and cyst recurrence; the recurrence rate for cysts 
is $>50 \%$ with this treatment [5]. Surgical excision is also associated with a recurrence rate of up to $39 \%$ [6]. Furthermore, there is a risk of treatment failure or adverse effects, such as increased pain and stiffness, infection, scarring, and permanent or temporary neurological damage, after surgical excision [3]. Therefore, a sustainable treatment that suppresses their recurrence is desirable, and demands for improved conservative treatments remain high.

Korean medicine (KM) therapy has been recognized for its superior effectiveness in the management of ganglion cysts, including acupuncture, moxibustion, acupotomy (new type of noninvasive acupuncture or microsurgery using a small needlescalpel), electroacupuncture, and pharmacopuncture [3, 7-10]. In particular, Scolopendra pharmacopuncture (SP) combined with electroacupuncture was reported to significantly reduce the size of wrist ganglions and their related symptoms, such as pain and discomfort. This treatment also achieves low recurrence rates with shorter treatment duration [11]. However, previously conducted studies comprised only one or two case series and were not sufficient to support the potential benefits of SP and electroacupuncture. Accordingly, this study was performed to determine the effectiveness of SP and electroacupuncture in ganglion cyst treatment through by quantitatively analyzing our case series. Based on the superior quality of evidence obtained from real-world clinical practice, we tried to evaluate the efficacy of KM therapies for ganglion cysts and to present a safe and standardized treatment protocol.

\section{MATERIALS AND METHODS}

\section{Subjects}

We conducted a retrospective chart review of 20 patients with ganglion cysts who visited Shin Min-Seop Korean Medicine Clinic, Jeonju, South Korea between April 2016 and May 2017. We included patients with ganglion cysts involving joints or tendons (sheaths) (ICD-10: M67.4) (2-1), who received SP and electroacupuncture at least once a week. Patients who understood the treatment and agreed to share their treatment information with research efforts were included. We excluded patients for whom the complete data records were not available, such as patients who discontinued treatment less than twice or those who had undergone surgery during the treatment regimen.

The research was approved by the Institutional Review
Board of the Korea Institute of Oriental Medicine (I-1708/001005-01). Written informed consent was obtained from each patient.

\section{Treatment}

The patients received SP and electroacupuncture as the main intervention. Both treatments were administered once per week on the same day by Korean Medicine doctors with 10 years of clinical experience.

Approximately $0.5 \mathrm{~mL}$ of SP solution was administered into the ganglion cysts using a sterile hypodermic syringe (30-31 gauge, 1.0 cc; DM Medicrat, Dongbang, Seoul, Korea). All SP solutions were prepared in a laboratory at the Korean Pharmacopuncture Institute, in accordance with Korea Good Manufacturing Practice, under the supervision of a Korean Medicine Doctor [12].

Electroacupuncture (CELLMAC, STN-100; Gyeonggi-do, Korea) was also performed in some patients after SP injection. Acupuncture needles with $0.25-\mathrm{mm}$ diameter and 30mm length were used (Dong Bang; Gyeonggi-do, Korea). The acupuncture needle was inserted through the ganglion cyst, with the tip of the needle targeting the center of the cyst. After needle insertion, a $2-\mathrm{Hz}$ current was applied for $12 \mathrm{~min}$ for electrostimulation.

As additional KM therapies to SP and electroacupuncture, acupuncture treatments tailored to the individual patients were performed according to the standard KM theory of pattern identification.

\section{Data collection and analysis}

The primary outcome was the size of the ganglion cyst; the diameter of the largest cyst was measured by ultrasound (SA6000 , Medison, South Korea) because the mere presence of ganglion cysts causes dissatisfaction and motivates patients to seek treatment, even in the absence of direct clinical symptoms [4]. The ultrasound images; Visual Analogue Scale (VAS) score for pain; Korean version of the disabilities of arm, shoulder, and hand (K-DASH) score [13]; Korean version of the patient-rated wrist evaluation (K-PRWE) score [14]; and recurrences as assessed by telephone calls were also evaluated.

All statistical analyses were performed using R- 3.5.2 for Windows. Continuous variables are expressed as means \pm standard deviations (SDs). Changes in the cyst diameter, VAS scores 
for pain, and questionnaire scores between the first and last visit were evaluated using paired t-tests.

\section{RESULTS}

Among the 31 patients, 20 who met the eligibility criteria were included in this study. The clinical characteristics of the eligible patients are summarized in Table 1. The mean duration of treatment was $37.2 \pm 41.01$ (median: 22) days and the number of treatment sessions was $3.95 \pm 1.67$ (median: 4).

The cyst diameter significantly decreased from $13.61 \pm 6.41$ $\mathrm{mm}$ to $5.15 \pm 6.18 \mathrm{~mm}(\mathrm{p}<0.001)$ at the last visit after treat-

Table 1. Clinical characteristics of 20 patients with ganglion cysts

\begin{tabular}{lc}
\hline Sex (M/F) & $9 / 11$ \\
Lesion site & 18 \\
Wrist & 2 \\
Ankle & \\
Consistency of the lesion & 7 \\
Hard & 2 \\
Soft & 11 \\
Not assessed & $37.2 \pm 41.01$ \\
\hline Treatment periods (days)
\end{tabular}

ment; the mean difference in size was $8.46 \mathrm{~mm}$ (95\% confidence interval [CI]: 6.25-10.66 mm; Table 2).

The pain VAS scores significantly reduced from $1.31 \pm 1.77$ to $0.41 \pm 0.33 \mathrm{~mm}(\mathrm{p}=0.021)$ after the last visit for treatment and symptom questionnaire scores showed significantly decreased scales as well in both the K-DASH and K-PRWE; the K-DASH score decreased from $8.97 \pm 12.66$ to $2.21 \pm 7.39$ with mean difference of 6.76 ( $\mathrm{p}=0.016$; 95\% CI: $1.45-12.08 \mathrm{~mm}$; Table 2), and the K-PRWE score decreased from $11.37 \pm 4.48$ to $9.1 \pm 3.67$ ( $\mathrm{p}=0.046$; 95\% CI: 0.05-4.48 mm; Table 2).

Gross and ultrasound findings before and after treatment for two representative patients are shown in Fig. 1 and 2. No recurrences were reported during the follow-up period, except for eight patients who were not accessible. The median followup period was $13 \pm 39.01$ days. Four patients experienced mild local adverse events at the site of injection. Two patients developed rash, one developed itching, and one developed a swelling. The swelling diminished within 1 day, while itching resolved after 19 days. The rash disappeared after 12 days in one patient and 10 days in the other.

\section{DISCUSSION}

This study was conducted to verify the effectiveness of SP

Table 2. Assessment of outcome measures

\begin{tabular}{lccccc}
\hline \multicolumn{1}{c}{ Variables } & Sample & Before treatment & After treatment & Mean difference $(95 \%$ Cl) & $p$-value \\
\hline Cyst diameter cyst $(\mathrm{mm})$ & 20 & $13.61 \pm 6.41$ & $5.15 \pm 6.18$ & $8.46(6.25-10.66)$ & $0.90(0.15-1.66)$ \\
VAS for pain & 20 & $1.31 \pm 1.77$ & $0.41 \pm 0.33$ & 0.001 \\
K-DASH score & 17 & $8.97 \pm 12.66$ & $2.21 \pm 7.39$ & $6.76(1.45-12.08)$ & 0.021 \\
K-PRWE score & 15 & $11.37 \pm 4.48$ & $9.1 \pm 3.67$ & $2.27(0.05-4.48)$ & 0.046 \\
\hline
\end{tabular}

VAS, visual analog scale; ROM, range of motion; K-DASH, Korean version of the disabilities of arm, shoulder and hand (K-DASH); K-PRWE, Korean version of the patient-rated wrist evaluation.
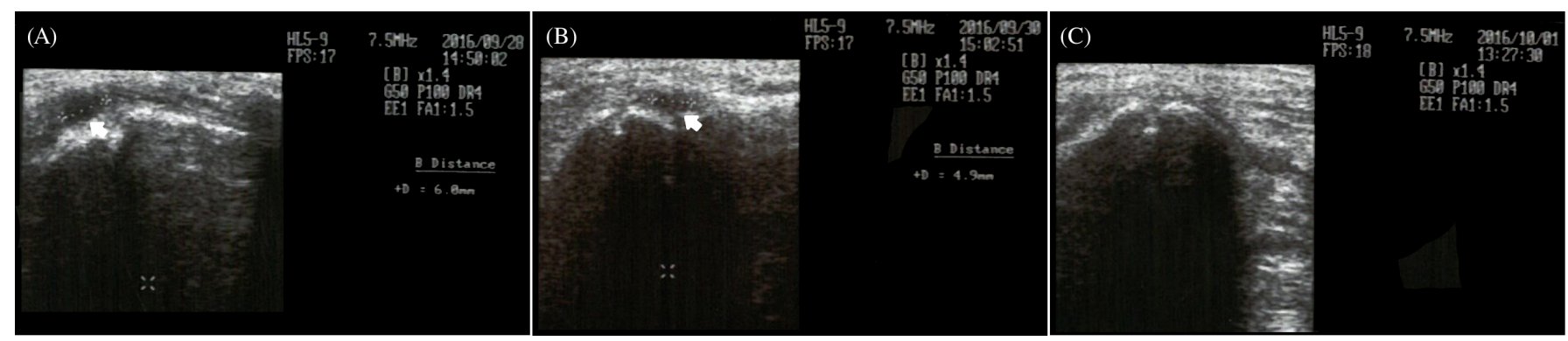

Figure 1. Ultrasound image before and after treatment for Case 1. The patient received an injection of $0.5 \mathrm{~mL}$ of Scolopendra pharmacopuncture solution in the ganglion cyst. (A-C) Changes in the ultrasound findings (white arrows). The diameter decreased from $6 \mathrm{~mm}$ before treatment to $4.9 \mathrm{~mm}$ on the day after treatment, and the cyst disappeared at 4 days after treatment. 


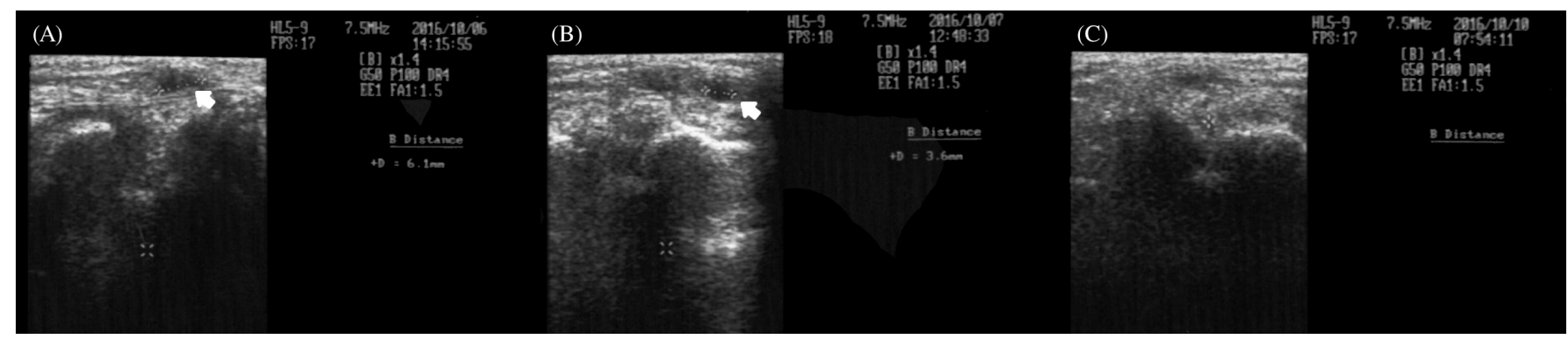

Figure 2. Ultrasound image before and after treatment for Case 2. The patient received an injection of $0.5 \mathrm{~mL}$ of Scolopendra pharmacopuncture solution in the ganglion cyst. (A-C) Changes in the ultrasound findings (white arrows). The diameter decreased from $6.1 \mathrm{~mm}$ before treatment to $3.6 \mathrm{~mm}$ on the day after treatment, and the cyst disappeared at 4 days after treatment.

combined with electroacupuncture for ganglion cysts. All patients showed improvement of ganglion cysts with no recurrence and a low complication rate. The size of the ganglion cysts exhibited a significant decrease after treatment with SP combined with electroacupuncture. The severity of pain decreased after treatment. Moreover, recurrence was not observed in any of the patients.

According to a systematic review [1], open surgical excision has a mean recurrence rate of $21 \%$, while aspiration has a recurrence rate of 59\%. Hence, SP combined with electroacupuncture may be more useful than aspiration and surgical excision in the treatment of ganglion cysts. Even though the incidence of adverse events in the present study was approximately $20 \%$, the events were mild, including mild rash, itching, and swelling at the injection site that resolved within a few days. The adverse events in treatment with SP combined with electroacupuncture are milder than those observed with aspiration and surgical excision, which can include nerve injury, artery laceration, persistent pain, and limitation of function [15].

Ganglion cyst is categorized as a phlegm nodule in Korean Medicine. It is thought to be formed by a combination of phlegm and static blood under the conditions of phlegm dampness; i.e., stagnant blood cannot flow for a long time [16]. Scolopendra is considered to dispel wind to free the collateral vessels, settle convulsive disease, relieve pain, and detoxify [11]. SP is expected to show complex effects that include the pharmacological action of Scolopendras and the effect of physical stimulation of the meridian point; hence, it has been used for treating joint pain and nerve entrapment syndrome [11, 17]. In a previous study [18], SP improved sciatic functional index in walking tract analysis and suppressed the pain severity in sciatic crushed nerve injury in rats. It suppressed COX-2 and TNF- $\alpha$ expression and enhanced the neurofilament expression in sci- atic crushed nerve injury. The findings of this study suggest the effectiveness of SP in the treatment of ganglion cysts.

Electroacupuncture may be beneficial in the treatment of ganglion cyst of the wrist, but only case series have been conducted to assess its efficacy $[3,7,8]$. Electroacupuncture blocks pain by activating a variety of bioactive chemicals through peripheral, spinal, and supraspinal mechanisms. These include opioids, which desensitize peripheral nociceptors and reduce proinflammatory cytokines peripherally and in the spinal cord; and serotonin and norepinephrine, which decrease spinal Nmethyl-d-aspartate receptor subunit GluN1 phosphorylation [19].

Several limitations could have led to potential bias in our study. The sample size was small, and combined therapies were used. It is difficult to determine which therapy provided the greatest contribution. However, considering the difficulties in conducting clinical trials on pharmacopuncture, the effectiveness of SP and electroacupuncture was established by retrospective chart review. Within the above-mentioned limitations, this study suggests that SP combined with electroacupuncture can not only reduce the size of the ganglion cysts and accompanying pain, but also ameliorate functional and psychological deterioration, with a low complication rate. Further studies with large samples reflecting various types of lesions are necessary to confirm our results.

\section{CONCLUSION}

This study verified that combination treatment of SP and electroacupuncture may be effective in treating ganglion cysts, by reducing the size of cysts, severity of pain and related symptoms.

However, the small number of included cases and combined 
other therapies remain a limitation of this study. Further prospective studies with large population are needed to clarify the effect of SP and electroacupuncture.

\section{ACKNOWLEDGMENT}

This study was supported by Korea Institute of Oriental Medicine (KSN1522120).

\section{CONFLICT OF INTEREST}

The authors declare that there are no conflicts of interest.

\section{ORCID}

Seon Choe, https://orcid.org/0000-0001-9504-2956

Ui Min Jerng, https://orcid.org/0000-0002-1996-3793

Jeong Hwan Park, https://orcid.org/0000-0003-2697-6530

Sungha Kim, https://orcid.org/0000-0001-5542-3850

Sungchul Kim, https://orcid.org/0000-0001-9274-9243

Jinbok Lee, https://orcid.org/0000-0003-2725-4062

Jun-Hwan Lee, https://orcid.org/0000-0001-5730-6869

Minseop Shin, https://orcid.org/0000-0002-1698-8987

\section{REFERENCES}

1. Head L, Gencarelli JR, Allen M, Boyd KU. Wrist ganglion treatment: systematic review and meta-analysis. J Hand Surg Am. 2015;40(3):546-53.e8.

2. Memon F, Abbas R, Khan MA, Quraishy MS. Aspiration combined with seton insertion for treatment of wrist ganglions. Pak J Surg. 2007;23(3):205-7.

3. Woitzik E, Kissel J. Ganglion cyst of the foot treated with electroacupuncture: a case report. J Can Chiropr Assoc. 2013;57(4): 310-5.

4. Gregush RE, Habusta SF. Ganglion Cyst [Internet]. Treasure Island (FL): StatPearls Publishing; 2020 Jan [updated 2020 Aug 25; cited 2020 Aug 25]. Available from: https://www.ncbi.nlm. nih.gov/books/NBK470168/.

5. Latif A, Ansar A, Butt MQ. Treatment of ganglions; a five year experience. J Pak Med Assoc. 2014;64(11):1278-81.

6. Meena S, Gupta A. Dorsal wrist ganglion: current review of literature. J Clin Orthop Trauma. 2014;5(2):59-64.
7. Kim KM, Kang EY, Lee SH, Jung AY, Nam DH, Cheon JH. Therapeutic approach of wrist ganglion using electroacupuncture: two case reports. Ann Rehabil Med. 2014;38(3):415-20.

8. Tekeoğlu I, Doğan A. Electroacupuncture in the treatment of a ganglion of the wrist--a case report. Acupunct Med. 2006;24(1): 29-32.

9. Kim KH. Clinical study on wrist ganglion by newly transformed centro-square acupuncture-moxibustion. J Korean Acupunct Moxib Soc. 2000;17(4):113-9.

10. Park MY, Lim NR, Kim SH, Kim SC. Effect of ultrasoundguided acupotomy therpy on wrist ganglion. J Korean Acupunct Moxib Soc. 2010;27(3):167-72.

11. Shin MS, Lee JB, Song BY. Two case report on wrist ganglion treated with scolopendrid pharmacopuncture. J Pharmacopuncture. 2010;13(2):139-43.

12. Cho G, Han K, Yoon J. Stability test and quantitative and qualitative analyses of the amino acids in pharmacopuncture extracted from Scolopendra subspinipes mutilans. J Pharmacopuncture. 2015;18(1):44-55.

13. Lee JY, Lim JY, Oh JH, Ko YM. Cross-cultural adaptation and clinical evaluation of a Korean version of the disabilities of arm, shoulder, and hand outcome questionnaire (K-DASH). J Shoulder Elbow Surg. 2008;17(4):570-4.

14. Kim JK, Kang JS. Evaluation of the Korean version of the patient-rated wrist evaluation. J Hand Ther. 2013;26(3):238-43; quiz 244.

15. Korkmaz M, Ozturk H, Amanvermez Senarslan D, Erdogan Y. Aspiration and methylprednisolone injection to the cavity with IV cannula needle in the treatment of volar wrist ganglia: new technique. Pak J Med Sci. 2013;29(1):103-6.

16. Won JH, Kim YJ, Ahn HD, Woo CH. Reviewing research of Korean medical treatment of wrist ganglion. J Korean Med Rehabil. 2014;24(4):111-6.

17. Son I, Lee S, Kim D, Jeong H, Cho SH, Lee EY, et al. A pilot study on single-dose toxicity testing of scolopendrid pharmacopuncture in sprague-dawley rats. J Pharmacopuncture. 2014; 17(2):57-66.

18. Lee GJ, Song YK, Lim HH. Effect of bee venom pharmacopuncture and scolopendra subspinipes pharmacopuncture on functional recovery and anti-inflammation after sciatic crushed nerve injury in rats. J Oriental Rehab Med. 2013;23(2):17-31.

19. Zhang R, Lao L, Ren K, Berman BM. Mechanisms of acupuncture-electroacupuncture on persistent pain. Anesthesiology. 2014;120(2):482-503. 